\title{
Functional Evaluation of Upper Urinary Tract with Diuretic Mercaptoacetyltriglycine Renal Scans in Patients with Benign Prostatic Obstruction before and after Surgical Intervention: A Pilot Study
}

\author{
Sung Yong Cho, ${ }^{1}$ Kyungtae Ko, ${ }^{2}$ Kyo Chul Koo, ${ }^{3}$ Hyung Joon Kim, ${ }^{4}$ Woo Jin Bang, \\ Min Soo Choo, ${ }^{6}$ Sang Hyub Lee, ${ }^{7}$ Young Eun Yoon, ${ }^{8}$ Wonho Jung ${ }^{(D)},{ }^{9}$ Jae Young Choi, ${ }^{10}$ \\ Dong Sup Lee $\mathbb{D}^{11}{ }^{11}$ and Young Endourological Study (YES) Group ${ }^{12}$
}

${ }^{1}$ Department of Urology, Seoul National University Hospital, Seoul, Republic of Korea

${ }^{2}$ Department of Urology, Kangdong Sacred Heart Hospital, Hallym University, Seoul, Republic of Korea

${ }^{3}$ Department of Urology, Gangnam Severance Hospital, Seoul, Republic of Korea

${ }^{4}$ Department of Urology, Konyang University Hospital, Daejeon, Republic of Korea

${ }^{5}$ Department of Urology, Hallym Sacred Heart Hospital, Hallym University, Anyang, Republic of Korea

${ }^{6}$ Department of Urology, Dongtan Sacred Heart Hospital, Hallym University, Hwaseong, Republic of Korea

${ }^{7}$ Department of Urology, KyungHee University Medical Center, Seoul, Republic of Korea

${ }^{8}$ Department of Urology, Hanyang University Hospital, Seoul, Republic of Korea

${ }^{9}$ Department of Urology, Dongsan Medical Center, Keimyung University School of Medicine, Daegu, Republic of Korea

${ }^{10}$ Department of Urology, Yeungnam University Medical Center, College of Medicine, Yeungnam University,

Daegu, Republic of Korea

${ }^{11}$ Department of Urology, St. Vincent's Hospital, The Catholic University of Korea, Suwon, Republic of Korea

${ }^{12}$ Endoluminal \& Technology, Seoul, Republic of Korea

Correspondence should be addressed to Dong Sup Lee; lds@catholic.ac.kr

Received 12 February 2020; Revised 29 June 2020; Accepted 4 July 2020; Published 10 August 2020

Academic Editor: Yujiang Fang

Copyright $\odot 2020$ Sung Yong Cho et al. This is an open access article distributed under the Creative Commons Attribution License, which permits unrestricted use, distribution, and reproduction in any medium, provided the original work is properly cited.

Introduction. We investigated which benign prostatic hyperplasia-related lower urinary parameters are related to upper urinary tract obstruction and whether transurethral prostatectomy could improve upper urinary tract obstruction. Materials and Methods. Patients with prostate size over $30 \mathrm{~g}$ and urodynamically proven bladder outlet obstruction were enrolled in this prospective observational study. Bladder wall thickness and prostate size were measured by ultrasonography. A urodynamic study with laboratory tests including serum creatinine, prostate-specific antigen, and urinalysis was performed. Finally, a diuretic scintigraphy using mercaptoacetyltriglycine was performed. Tests except the urodynamic evaluation were repeated after transurethral prostatectomy. Results. In total, 24 patients were enrolled, and 19 patients completed the present study. The mean values of age (yrs), prostate size $(\mathrm{mL})$, bladder thickness $(\mathrm{mm})$, bladder compliance $(\Delta \mathrm{mL} / \Delta \mathrm{pr})$, and the bladder outlet obstruction index were $68.42 \pm 8.25,72.29 \pm 32.78,4.42 \pm 1.14,50.17 \pm 32.15$, and $82.11 \pm 34.68$, respectively. The mean $\mathrm{T}_{1 / 2}$ (min) was $17.51 \pm 16.34$ on the left side and $15.30 \pm 11.96$ on the right side. Statistical analysis showed that bladder compliance and bladder thickness were preoperatively related to upper urinary tract obstruction ( $p=0.001$ and $p=0.007$, respectively). Diuretic mercaptoacetyltriglycine scan in 19 patients showed improvement 6 months after prostate surgery. Clinically significant proteinuria was associated with upper urinary tract obstruction, and proteinuria was also improved after prostate surgery. Conclusion. Storage-phase bladder dysfunction could be a reliable urodynamic factor for the indication of upper urinary tract obstruction in patients with benign prostatic hyperplasia, and upper urinary tract obstruction with subsequent kidney damage could be improved by surgical decompression of benign prostatic obstruction. 


\section{Introduction}

Benign prostatic hyperplasia (BPH) is a prevalent disease in which approximately $50 \%$ of men aged 60 have lower urinary tract symptoms [1]. More importantly, $\mathrm{BPH}$ is a progressive disease [2] that may result in chronic renal insufficiency as one of the final stages of BPH progression [3]. Nevertheless, it is still unclear whether $\mathrm{BPH}$ is a risk factor for chronic kidney disease (CKD) regarding clinical evidence $[4,5]$. If $\mathrm{BPH}$ causes chronic obstructive uropathy and eventually leads to renal insufficiency, there may be causative anatomical factors or urodynamic factors that could delay urinary drainage from the kidney to the urinary bladder.

With respect to the functional evaluation of upper urinary tract obstruction, the diuretic mercaptoacetyltriglycine $\left({ }^{99 \mathrm{~m}} \mathrm{Tc}-\mathrm{MAG} 3\right)$ renal scan (hereafter referred to as MAG3) is a feasible imaging tool to evaluate upper urinary tract obstruction [6]. To our knowledge, no clinical study has investigated the relationship of BPH-related lower urinary tract factors and upper urinary tract parameters with MAG3 in patients with BPH. Therefore, the authors conducted a pilot study to investigate whether benign prostatic obstruction (BPO)-related lower urinary tract parameters are related to upper urinary tract obstruction and whether the decompression of BPO could ameliorate upper urinary tract obstruction.

\section{Materials and Methods}

2.1. Study Design and the Enrollment of Patients. Patients who complained of lower urinary tract symptoms despite alpha blocker medication were prospectively enrolled from July 2016 to June 2019. Before enrollment, ultrasound (for kidney, bladder, and prostate; bladder thickness was measured at the bladder dome when patients felt the desire to voiding desire), a urodynamic study, and laboratory tests including PSA, serum creatinine with estimated glomerular filtration rate (eGFR) [7], and urinalysis were performed.

Patients with a PSA value of $4.0(\mathrm{ng} / \mathrm{mL})$ or higher underwent ultrasound-guided transrectal prostate biopsy to exclude prostate cancer. When eGFR was $<60 \mathrm{ml} / \mathrm{min} / 1.73 \mathrm{~m}^{2}$ or when clinically significant proteinuria was observed, the tests were repeated 3 months later to confirm CKD according to the universal protocol [8], and then, the better result was employed. Laboratory tests were repeated at 6 months after transurethral prostate surgery.

International Prostate Symptom Score (IPSS) questionnaire scores evaluated the following seven items were assessed: bladder emptying, frequency, intermittency, urgency, weak stream, straining, and nocturia [9]. The score from each item ranged from 0 to 5 . The IPSS questionnaire was repeated 6 months after transurethral prostate surgery.

For noninvasive uroflowmetry, patients voided freely without catheterization. At that time, we measured and recorded the maximum flow rate (Qmax) and postvoid residual urine volume (PVR). The PVR was measured using a Biocon 500 ultrasound scanner (Medline Industries, Inc., Mundelein, Illinois, USA). Filling and voiding cystometry was performed, including parameters such as bladder com- pliance $(\Delta \mathrm{mL} / \Delta \mathrm{pr})$, the presence of involuntary detrusor contraction (IDC), the bladder outlet obstruction index (BOOI), Schäfer grades, and the bladder contractility index (BCI) [10]. A urodynamic study was performed only before surgery. Instead, patients were followed up with uroflowmetry 6 months postoperatively.

Patients who met all the following inclusion criteria (suggesting BPO) were enrolled in the present study: (1) those with evidence of $\mathrm{BOO}$ (BOOI $\geq 40$ or Schäfer grade $\geq 2$ ); and (2) those with a prostate total volume $\geq 30 \mathrm{~mL}$ in ultrasonography [11-13]. Patients who had any one of the following were excluded from the present study: (1) acute urinary retention during the screening period, (2) urinary tract infection during the screening period, (3) ongoing malignancy, (4) uncontrolled diabetes mellitus (HbAlc >8.0), (5) uncontrolled hypertension (systolic blood pressure $>160 \mathrm{mmHg}$ ), (6) moderate or severe liver cirrhosis, (7) moderate or severe heart failure (NYHA class $\geq \mathrm{II}$ ), or (8) spinal cord disease such as myelopathy.

All patients enrolled in the study underwent diuretic MAG3. According to a previous investigation, 5 minutes was normal value of $\mathrm{T}_{1 / 2}$ in men aged 50 60 years [14]. The authors defined $\mathrm{T}_{1 / 2}=10$ minutes (time point when half of the ${ }^{99 \mathrm{~m}} \mathrm{Tc}$ substance is excreted) as the cut-off to distinguish whether the result of the renal scan was negative $(<10$ minutes) or positive ( $\geq 10$ minutes), because (1) BPO patients with $\mathrm{T}_{1 / 2}>20$ minutes would be very rare unless the obstruction was very severe and (2) 10 minutes is close to the median $\mathrm{T}_{1 / 2}$ value preoperatively. If the result was positive, the authors suggested it as "potential obstruction." The MAG3 was postoperatively reevaluated at 6 months. We had also presented the perioperative change with the analysis of the continuous value of MAG3 to compensate the limitation of using the arbitrary cut-off level. Comparison between the mean preoperative MAG3 value and the mean postoperative MAG3 value and correlation between the change of MAG3 value and the other continuous values were performed.

To the best of our knowledge, the effect of transurethral prostate surgery for BPO on upper urinary tract drainage has never been reported. The authors analyzed the effect of the transurethral prostate surgery for BPO on the drainage condition as follows. If a $25 \%$ or more decrease (at least 1 kidney) was observed in the $\mathrm{T}_{1 / 2}$ of the postoperative result compared to the $\mathrm{T}_{1 / 2}$ of the preoperative result, the authors assumed that the upper urinary tract drainage was "relatively" enhanced; furthermore, if a $50 \%$ or more decrease (at least 1 kidney) was identified in the $T_{1 / 2}$ of MAG3, the authors assumed the upper urinary tract drainage was "definitely" enhanced.

2.2. Transurethral Prostate Surgery. Transurethral prostate surgery was performed by surgeons with experience in over 50 cases of holmium laser enucleation of the prostate. Laser energy for enucleation of an adenoma was generally $2.0 \mathrm{~J}$ with $20 \mathrm{~Hz}$. The other techniques were similar to those in previously reported methods [15].

2.3. Statistics. The authors hypothesized that the upper urinary tract drainage measured by MAG3 could be enhanced 
6 months after transurethral surgery in BPO. As a pilot study, two-tailed sign test (binominal test) was used with a beta error of 0.2 , an alpha error of 0.05 , and an effect size as 0.3 to determine that the target sample size was 20 patients. Assuming a drop-out rate of approximately $20 \%$, we enrolled 24 patients in the present study.

The Mann-Whitney $U$ test and Wilcoxon test were used to check the difference before and 6 months after operation. The chi-squared test or Fisher's exact test was used to analyze binominal relationships between the groups. Spearman's test was used to investigate the association among the parameters. All statistical analyses were performed with the statistical package IBM-SPSS for Windows (Version 23.0; IBM Crop, Armonk, NY, USA). $p<0.05$ was regarded as statistically significant.

\section{Results}

In total, 24 patients were enrolled in the present study. Five patients were excluded due to the postoperative confirmation of pathologically-proven prostate cancer in 3 patients, loss to follow-up in one patient, and cervical myelopathy discovered during follow-up in 1 patient. In total, 19 patients completed the study.

The mean age was $68.42 \pm 8.25$ years, and the mean BMI was $23.83 \pm 1.66 \mathrm{~kg} / \mathrm{m}^{2}$. The baseline patient characteristics are shown in Table 1.

Among 19 patients with $\mathrm{BPO}$, a preoperative $\mathrm{T}_{1 / 2}$ of MAG3 over 10 minutes (potential obstruction) was observed in 13 patients (21 kidneys). Preoperative potential obstruction in at least 1 kidney was associated with clinically significant proteinuria (Spearman's rho $=0.482, p=0.036$ ). The associations between preoperative potential obstruction in at least 1 kidney and other factors are shown in Table 2.

At 6 months after transurethral prostate surgery, potential obstruction remained in 6 patients ( 9 kidneys). Based on the aforementioned criteria, "relative" improvement of upper urinary tract drainage was observed in 16 patients (26 kidneys), and "definite" improvement was seen in 4 patients (6 kidneys).

All laboratory tests, including prostate-specific antigen, eGFR, and urinary proteinuria, were improved after transurethral prostate surgery. The mean prostate-specific antigen level decreased from $6.67 \pm 4.52$ to $1.55 \pm 1.44$. The mean QoL scores in the IPSS questionnaire improved from 4.78 \pm 0.73 to $1.11 \pm 0.76$. The other results from the comparison between preoperative values and postoperative values are shown in Figure 1. Although voiding volume did not increase significantly, Qmax was remarkably improved, and postvoid residual volume decreased obviously.

As lower urinary factors such as bladder compliance and bladder thickness were related to the $T_{1 / 2}$ of MAG3, the authors investigated the likelihood of improvement in the $\mathrm{T}_{1 / 2}$ of MAG3 based on bladder compliance and bladder thickness. Because most patients with preoperatively good compliance (less thickened bladder) had a $\mathrm{T}_{1 / 2}$ of MAG3 under 10 minutes, the value did not change very much at 6 months after operation, whereas BPO patients who had preoperatively low compliance with thickened bladder and a
TABle 1: Patients' baseline characteristics $(n=19)$.

\begin{tabular}{|c|c|}
\hline Age (years) & $68.42 \pm 8.25$ \\
\hline Body mass index $\left(\mathrm{kg} / \mathrm{m}^{2}\right)$ & $23.83 \pm 1.66$ \\
\hline Prostate-specific antigen $(\mathrm{ng} / \mathrm{mL})$ & $6.67 \pm 4.52$ \\
\hline Serum creatinine $(\mathrm{mg} / \mathrm{dL})$ & $1.25 \pm 0.90$ \\
\hline eGFR $\left(\mathrm{mL} / \mathrm{min} / 1.73 \mathrm{~m}^{2}\right)$ & $71.68 \pm 19.43$ \\
\hline \multicolumn{2}{|l|}{ Proteinuria } \\
\hline Trace or more & $15 / 19(78.9 \%)$ \\
\hline+1 or more & $3 / 19(15.8 \%)$ \\
\hline \multicolumn{2}{|l|}{ Uroflowmetry parameters } \\
\hline $\mathrm{Qmax}(\mathrm{mL} / \mathrm{sec})$ & $8.51 \pm 4.91$ \\
\hline Voiding volume (mL) & $181.11 \pm 96.74$ \\
\hline Postvoid residual volume (mL) & $98.53 \pm 89.92$ \\
\hline \multicolumn{2}{|l|}{ Invasive urodynamic parameters } \\
\hline Bladder compliance $(\Delta \mathrm{mL} / \Delta \mathrm{pr})$ & $50.17 \pm 32.15$ \\
\hline Involuntary detrusor contraction & $2 / 19(10.5 \%)$ \\
\hline Bladder outlet obstruction index & $82.11 \pm 34.68$ \\
\hline Schäfer grades & $3.95 \pm 1.47$ \\
\hline Bladder contractility index & $114.45 \pm 40.24$ \\
\hline \multicolumn{2}{|l|}{ Anatomical factors } \\
\hline Prostate size $(\mathrm{mL})$ & $72.29 \pm 32.78$ \\
\hline Bladder thickness (mm) & $4.42 \pm 1.14$ \\
\hline IPSS total score & $22.44 \pm 7.51$ \\
\hline Storage symptom score & $8.72 \pm 3.88$ \\
\hline Obstructive symptom score & $13.72 \pm 4.03$ \\
\hline Quality of life score & $4.78 \pm 0.73$ \\
\hline \multicolumn{2}{|l|}{$\mathrm{T}_{1 / 2}$ of ${ }^{99 \mathrm{~m}} \mathrm{Tc}-\mathrm{MAG} 3$} \\
\hline Left side & $17.51 \pm 16.34$ \\
\hline Right side & $15.30 \pm 11.96$ \\
\hline $\mathrm{T}_{1 / 2}>10$ min at least one kidney & $13 / 19(68.4 \%)$ \\
\hline \multicolumn{2}{|l|}{ Underlying conditions } \\
\hline Diabetes mellitus & $4 / 19(21.1 \%)$ \\
\hline Hypertension & $7 / 19(36.8 \%)$ \\
\hline Smoking & $5 / 19(26.3 \%)$ \\
\hline Under $20 \mathrm{p}^{*} \mathrm{yrs}$ & $2 / 19(10.5 \%)$ \\
\hline Over $20 \mathrm{p}^{*} \mathrm{yrs}$ & $3 / 19(15.8 \%)$ \\
\hline Education (high school or more) & $13 / 19(68.4 \%)$ \\
\hline Recent symptom aggravation (year) & $1.33 \pm 1.09$ \\
\hline Previous history of acute urinary retention & $3 / 19(15.8 \%)$ \\
\hline
\end{tabular}

Parameters were expressed by the mean \pm standard deviation or frequency (\%). eGFR: estimated glomerular filtration rate; Qmax: maximal flow rate; IPSS: international prostate symptom score; MAG-3: diuretic mercaptoacetyltriglycine.

tendency for delay in $\mathrm{T}_{1 / 2}$ had much room for improvement in the $\mathrm{T}_{1 / 2}$ of MAG3 (Figures 2 and 3). This observation means that transurethral prostate surgery in BPO patients with low compliance and/or thickened bladder may be beneficial to improve upper urinary tract drainage. 
Clinically, significant proteinuria remained in 4 patients (preoperative proteinuria was found in 15 patients), and potential obstruction was still observed in 6 patients (preoperative potential obstruction was observed in 13 patients) at 6 months after the operation. In the correlation test, postoperative proteinuria was related not to upper urinary tract obstruction but to underlying diabetes mellitus (Table 3).

\section{Discussion}

The interesting finding of this study is that filling-phase bladder dysfunction, such as low compliance, could explain the occurrence of obstruction in the MAG3 in the present cohort. A similar study was conducted with pediatric neurogenic bladders, where the authors emphasized low bladder compliance and bladder wall thickness as a predictor of renal damage [16]. Many clinical studies support the likelihood of an association between bladder wall thickness and BPO [17], describing that the measurement of bladder wall thickness has a median sensitivity of $82 \%$ and a specificity of $92 \%$ for the prediction of BOO. De Nunzio et al. found that detrusor overactivity was significantly associated with bladder wall thickness even in non-BPO patients [18]. Therefore, the thickened bladder is not an exclusive property of $\mathrm{BOO}$ (or BPO); rather, it may be more related to detrusor condition regardless of BPO. Consequently, chronic BOO secondary to $\mathrm{BPH}$ can be a nonneurogenic factor among various lower urinary tract conditions that thicken the urinary bladder wall [19].

The BOOI was not significantly associated with the status of the upper urinary tract drainage measured by the $T_{1 / 2}$ of MAG3. Instead, only bladder compliance was responsible for upper urinary tract drainage in the present study. Two cases of IDC also showed a delay in the $\mathrm{T}_{1 / 2}$ of MAG3. These findings indicated that $\mathrm{BOO}$ itself would not induce obstruction in the upper urinary tract, but the long-standing high intravesical pressure induced by storage-phase bladder dysfunction might be the reason for upper urinary tract obstruction. Although the number of patients included is small, the authors suggest that $\mathrm{BPH}$ might not be the direct cause of renal insufficiency and $\mathrm{BPH}$ may be one of the factors that may induce bladder dysfunction, such as low compliance, which can affect urine drainage. This may also mean that the transient elevation of intravesical pressure during voiding in patients with $\mathrm{BPH}$ or a high BOOI without abnormalities in the filling phase would not induce renal insufficiency. This finding is consistent with previous investigations with neurogenic bladder [20, 21].

Considering that PdetQmax is incorporated in the BCI and the BOOI, it is possible that detrusor contraction increased to overcome the large size of the prostate. Nevertheless, the BCI was also not related to the $\mathrm{T}_{1 / 2}$ of MAG3. Because evidence of $\mathrm{BOO}$ (with a BOOI of 40 or more) was a criterion for inclusion in the present study, the bladder contractility in the majority of patients was intact or relatively preserved. According to several suggested criteria for detrusor underactivity (DU) [22, 23], PdetQmax should be under $30 \mathrm{cmH}_{2} \mathrm{O}$. Therefore, no one could have had DU in this cohort. However, researchers should be aware that either
TABLE 2: Association between potential obstruction ${ }^{1}$ and other factors in preoperative data.

\begin{tabular}{lc}
\hline Upper tract factor & $0.482, p=0.036^{*}$ \\
\hline Clinically significant proteinuria & $0.083, p=0.736$ \\
eGFR & \\
Lower tract factor & $0.614, p=0.007^{*}$ \\
Bladder thickness & $-0.269, p=0.266$ \\
Prostate size & \\
Urodynamic factor & \\
$\quad$ Storage factors & $-0.310, p=0.196$ \\
$\quad$ Voiding volume & $-0.704, p=0.001^{*}$ \\
$\quad$ Bladder compliance & \\
Obstructive factors & $-0.021, p=0.933$ \\
$\quad$ Qmax & $-0.145, p=0.554$ \\
Postvoid residual volume & $0.041, p=0.866$ \\
$\quad$ Bladder outlet obstruction index & \\
Other factor & $-0.031, p=0.583$ \\
Bladder contractility index & \\
General factors & $-0.031, p=0.899$ \\
Age & $-0.165, p=0.499$ \\
Body mass index & $-0.205, p=0.401$ \\
Diabetes mellitus & $-0.049, p=0.841$ \\
Hypertension & $p<0.05{ }^{1}: p 0617$
\end{tabular}

Data were expressed by Spearman's rho with $p$ value. ${ }^{*} p<0.05 .{ }^{1}$ : potential obstruction means preoperative $\mathrm{T}_{1 / 2}$ of ${ }^{99 \mathrm{~m}} \mathrm{Tc}-\mathrm{MAG} 3>10$ minutes at least 1 kidney.

BPO without DU or impaired detrusor activity can cause chronic urinary retention (CUR) [24], and CUR can be associated with hydronephrosis [25]. CUR is a clinical definition (not by urodynamic study), and most of the literature indicates CUR as PVR over $300 \mathrm{~mL}$ [25]. In the present study, only 1 case of CUR was shown in a patient with a prostate size of $58.5 \mathrm{~mL}$, BOOI of 48 , BCI of 55, bladder compliance of 33 , and $\mathrm{T}_{1 / 2}$ of MAG3 over 10 minutes in the left kidney. It indicates that the patient had high-pressure CUR (BPO without DU) [26]. Therefore, one of the limitations of the present study is that the authors cannot determine whether DU in BPH patients (BPH with low-pressure CUR) could also indicate a connection between bladder compliance and a delay in the $\mathrm{T}_{1 / 2}$ of MAG3 in terms of CUR.

Prostate size was not associated with the $T_{1 / 2}$ of MAG3, as the BOOI and BCI were not related to the $\mathrm{T}_{1 / 2}$ of MAG3. Previous studies have already suggested that prostate size might not be related to the decrease in renal function [5, 27]. Consequently, the authors believe that prostate size and relevant functional parameters, such as the BOOI and/or BCI, could not directly influence the delay in urinary drainage from the upper urinary tract because the urinary bladder acts as a "buffer." In the case of a loss of flexibility of the buffer (low compliance) or of a fullness of the buffer (CUR), the endorgan (BPO) effect could develop. Therefore, preoperative urodynamic evaluation in $\mathrm{BPH}$ is very important. 


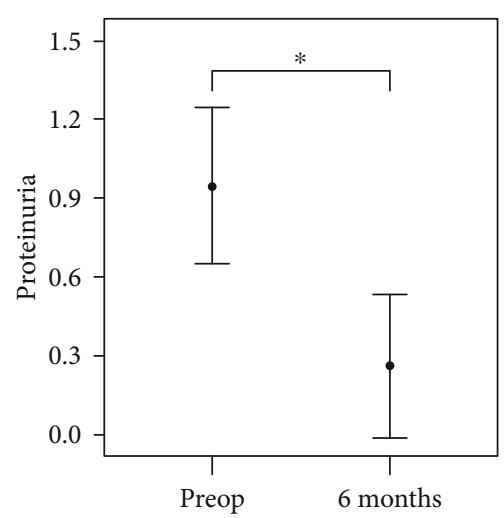

(a)

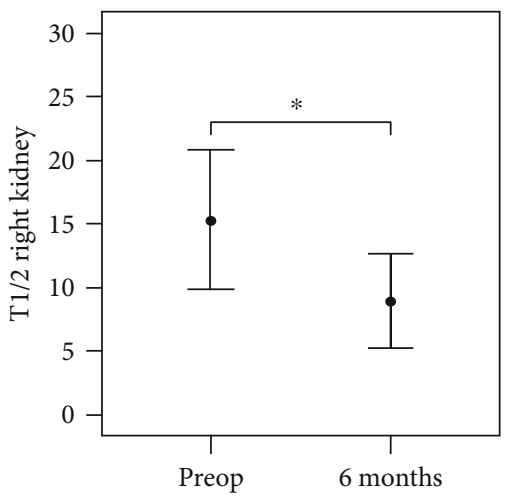

(d)

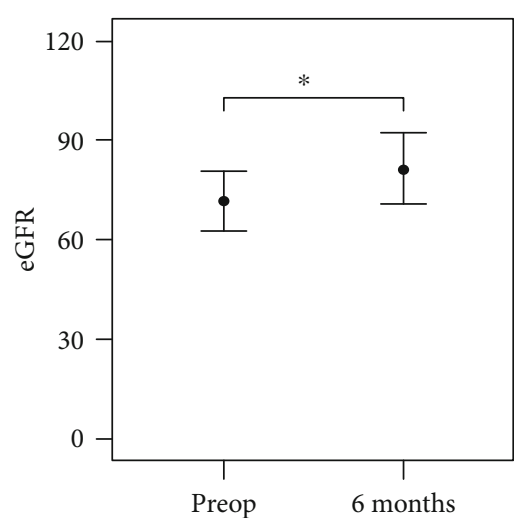

(b)

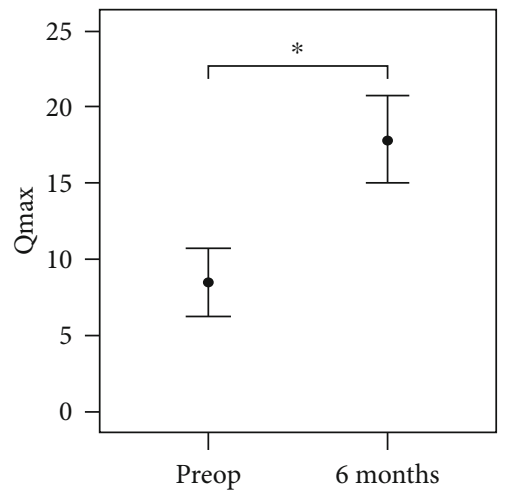

(e)

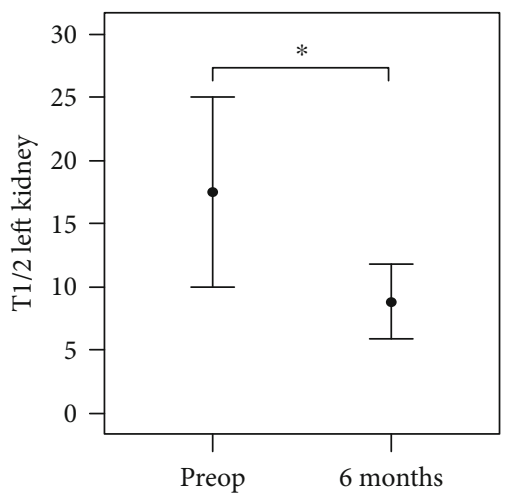

(c)

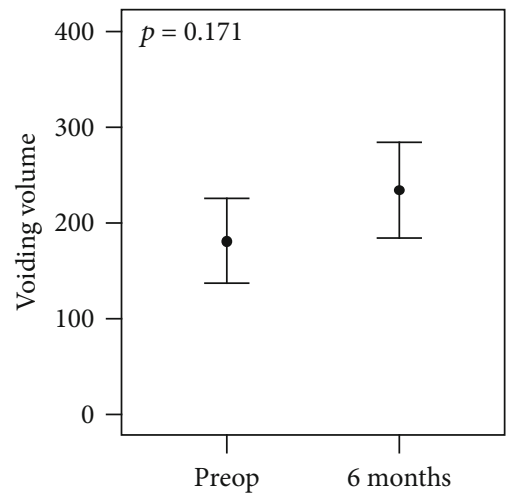

(f)

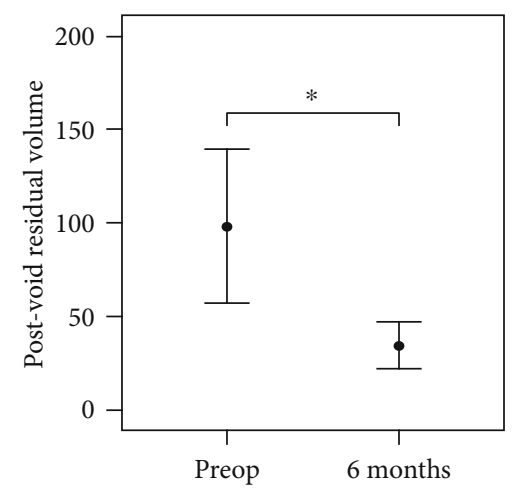

(g)

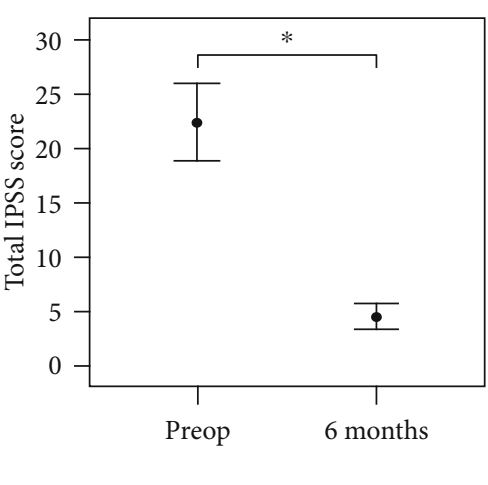

(h)

FIgURE 1: Changes in parameters before and at 6 months after the decompression of BPO. $(a, b)$ Renal function was improved. (c, $d)$ The $T_{1 / 2}$ of diuretic ${ }^{99 \mathrm{~m}} \mathrm{Tc}-\mathrm{MAG} 3$ was improved. (e-g) The mean values of Qmax, voiding volume, and PVR seemed to be enhanced, but voiding volume did not significantly increase. (h) The IPSS score was markedly improved. BPO: benign prostatic obstruction; PVR: postvoid residual urine volume; IPSS: international prostate symptom score. Asterisk $\left(^{*}\right)$ means $p<0.05$.

It was very interesting that preoperative proteinuria was associated with a delay in the $T_{1 / 2}$ of MAG3. A recent study showed that clinically significant proteinuria was more frequently identified in BPO patients with low bladder compliance [28], and the authors found that transurethral prostate surgery for those patients mitigated proteinuria and that the phenomenon was obvious in patients without underlying medical disease. Considering that proteinuria is a marker of ongoing renal damage [29], the present study revealed the probability that BPO with functionally low bladder compli- ance and an anatomically thickened bladder wall may delay the $T_{1 / 2}$ of MAG3, resulting in renal damage (proteinuria) even in patients without CUR.

The postoperative $T_{1 / 2}$ of MAG3 was not related to the remaining proteinuria at 6 months postoperatively, which indicated that underlying hypertension and/or DM might be more responsible for the remaining proteinuria (Table 3). Conversely, BPO could be involved in the pathophysiology of developing CKD. Considering CKD can also be multifactorial, we could not definitely rule out intrinsic 


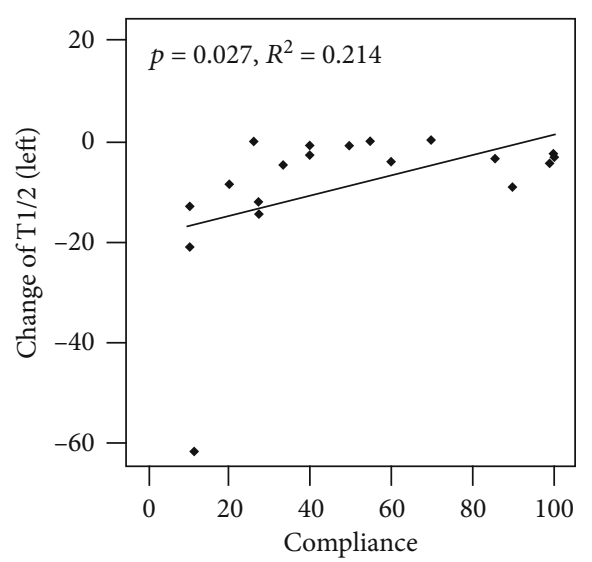

(a)

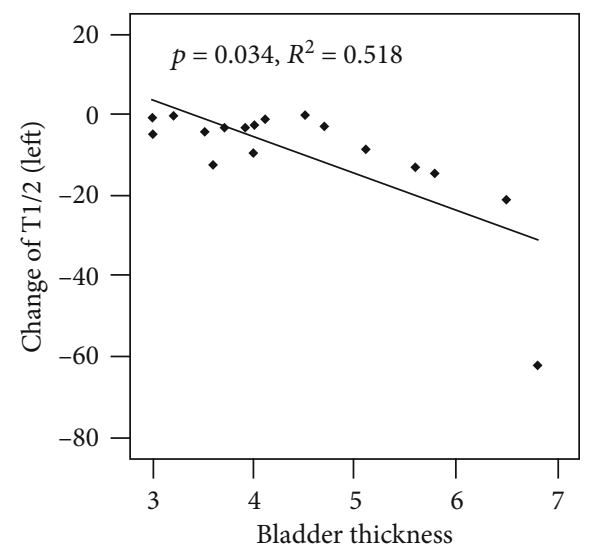

(c)

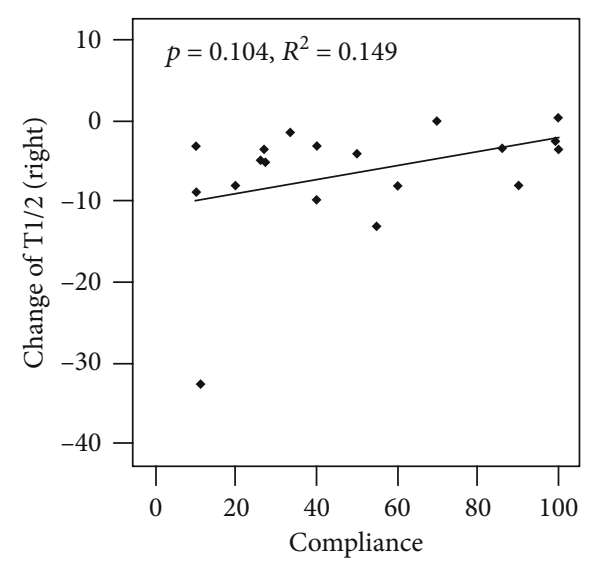

(b)

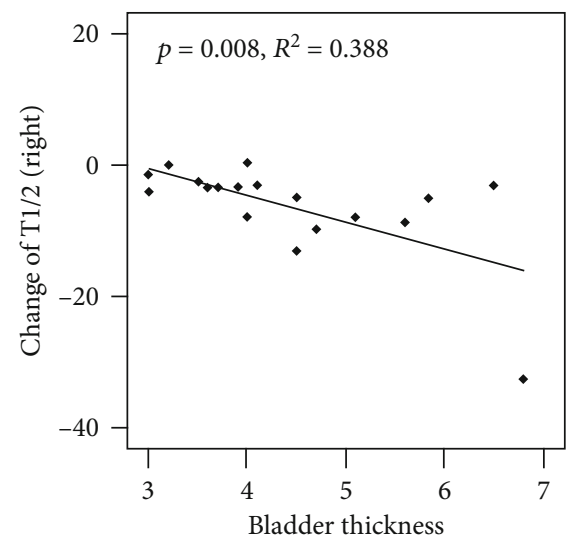

(d)

FIGURE 2: Changes in the $\mathrm{T}_{1 / 2}$ of the diuretic ${ }^{99 \mathrm{~m}} \mathrm{Tc}-\mathrm{MAG} 3$ scan according to preoperative bladder compliance and bladder thickness. (a, b) The preoperative bladder compliance was lower, and the change in the $\mathrm{T}_{1 / 2}$ of the diuretic ${ }^{99 \mathrm{~m}} \mathrm{Tc}-\mathrm{MAG} 3$ scan was greater. (c, $\mathrm{d}$ ) The preoperative bladder was thicker, and the change in the diuretic ${ }^{99 \mathrm{~m}} \mathrm{Tc}-\mathrm{MAG} 3 \mathrm{scan}$ was greater.

renal causes presenting proteinuria because we did not perform or recommend renal biopsy in the present cohort, which could be a limitation of the study. Nevertheless, in the present study, proteinuria, eGFR, and the $T_{1 / 2}$ of MAG3 were significantly improved after surgery. The authors think that the preoperative discordance between eGFR and $T_{1 / 2}$ of MAG3 might be due to the following reasons: (1) the baseline eGFRs were close-set one another; (2) individual baseline eGFR could be determined not only by the urinary drainage condition but also by various factors such as age and underlying diseases including hypertension and DM; and (3) the decrease in eGFR is a quite late response compared to the development of significant proteinuria [30].

Nomura et al. showed urodynamic results in patients who underwent transurethral prostate surgery. Half of those patients with detrusor overactivity improved 6 months after operation [31]. Furthermore, an interesting result was reported by Tkocz et al. that transurethral resection or incision of the prostate could sufficiently improve bladder compliance as well as remarkably decrease the incidence of detrusor overactivity unless the detrusor structure had irreversibly changed [32]. The absence of postoperative urodynamic analysis was a major limitation of the present study, but the preoperative relationship between the $T_{1 / 2}$ of MAG3 and bladder compliance and the improvement in the $\mathrm{T}_{1 / 2}$ of MAG3 could support our assumption for the improvement in postoperative bladder compliance.

Meanwhile, 5 alpha reductase inhibitors (5ARIs) can be used for pharmacological deobstruction for BPO. According to the early research conducted by Stoner who used finasteride for $\mathrm{BPH}$ treatment for 36 months, he found that the prostate volume was reduced by $27 \%$ compared to baseline (over $45 \mathrm{~mL}$ ), and Qmax was improved by $2.3 \mathrm{~mL} / \mathrm{s}$ [33]. Similarly, in CombaT study, even with 24 months of combination therapy (dutasteride plus tamsulosin), Qmax improved by $2.2 \sim 3.0 \mathrm{~mL} / \mathrm{s}$ [34]. Although pharmacological deobstruction using 5ARIs (with or without alpha 1 selective adrenoreceptor antagonist) could be beneficial to relieve the $\mathrm{BPO},(1)$ it takes too much time to observe the results, and (2) the change of parameters such as increase in Qmax and decrease in prostate volume may be tiny, compared to surgical decompression. Therefore, the pharmacological deobstruction seems to be inappropriate as a control group of a study evaluating the MAG3 parameter.

Finally, the present study contains severe forms of $\mathrm{BPH}$, with a mean bladder compliance, BOOI, prostate size, and 


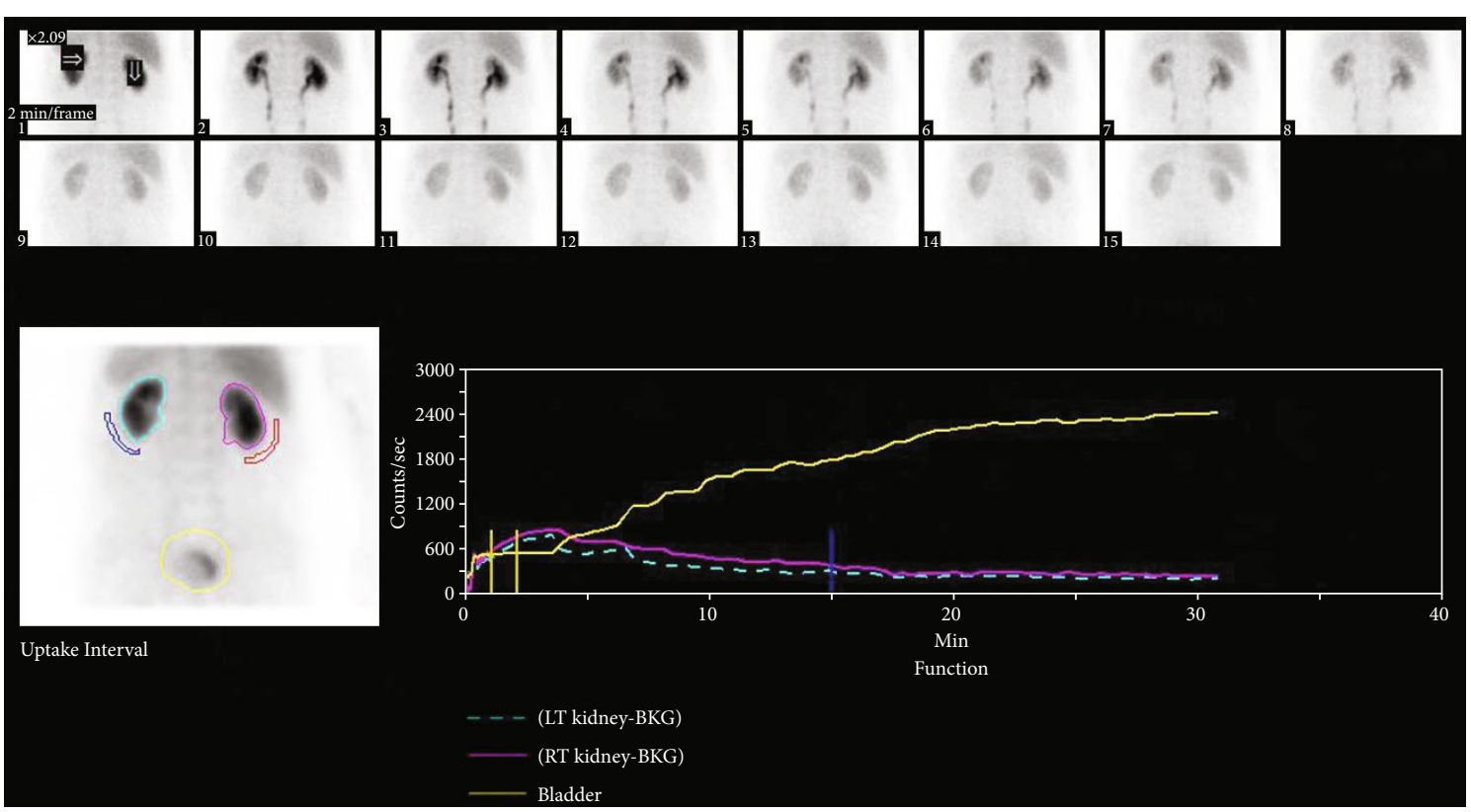

(a)

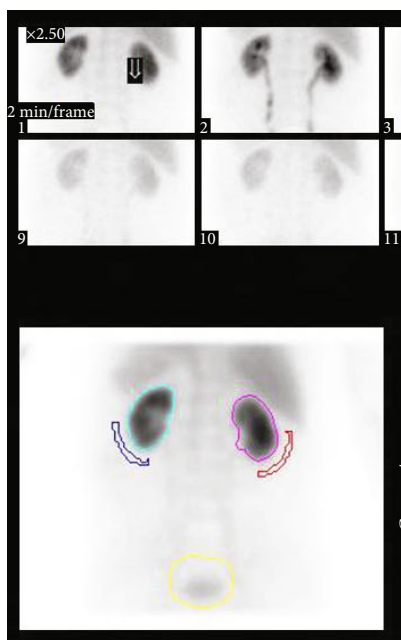

Uptake Interval

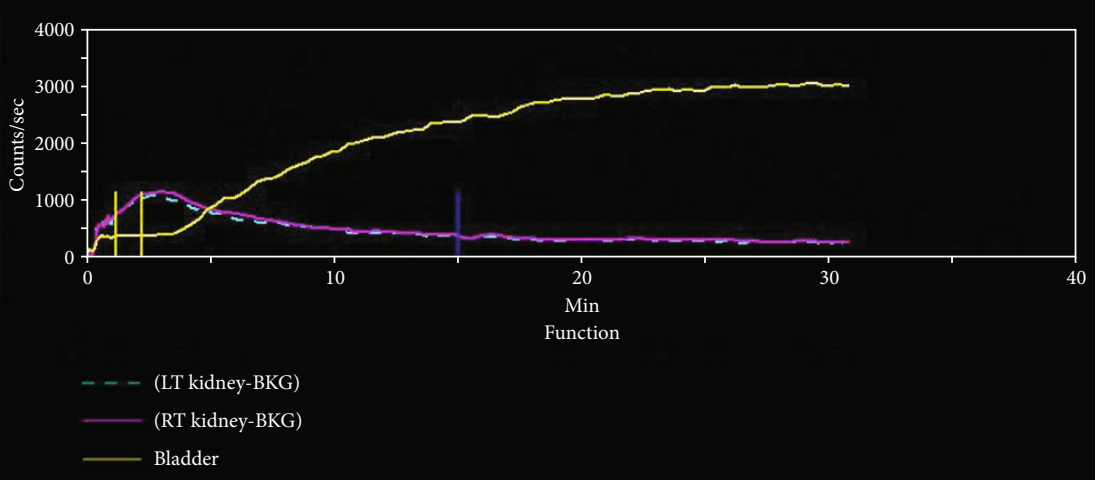

(b)

FIgure 3: Continued. 


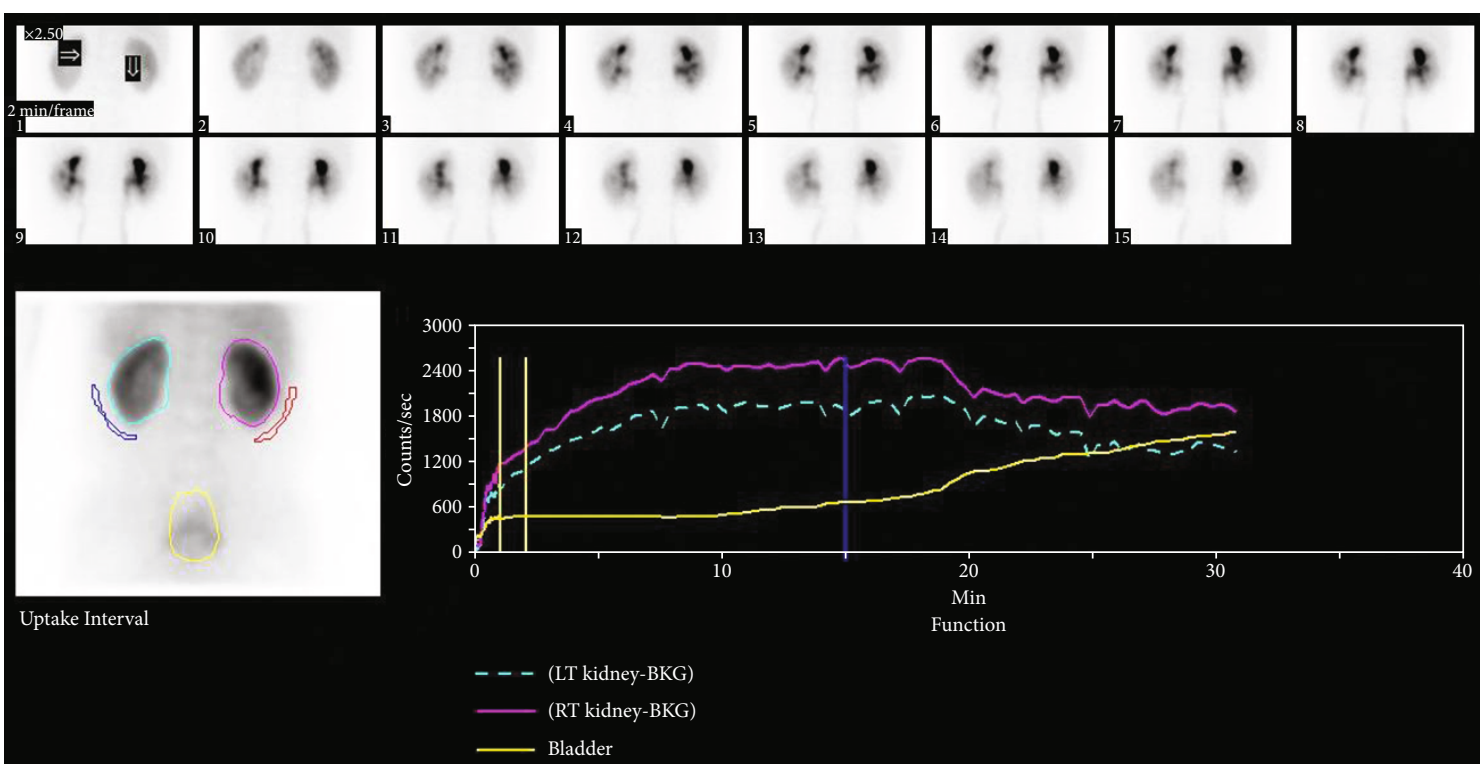

(c)

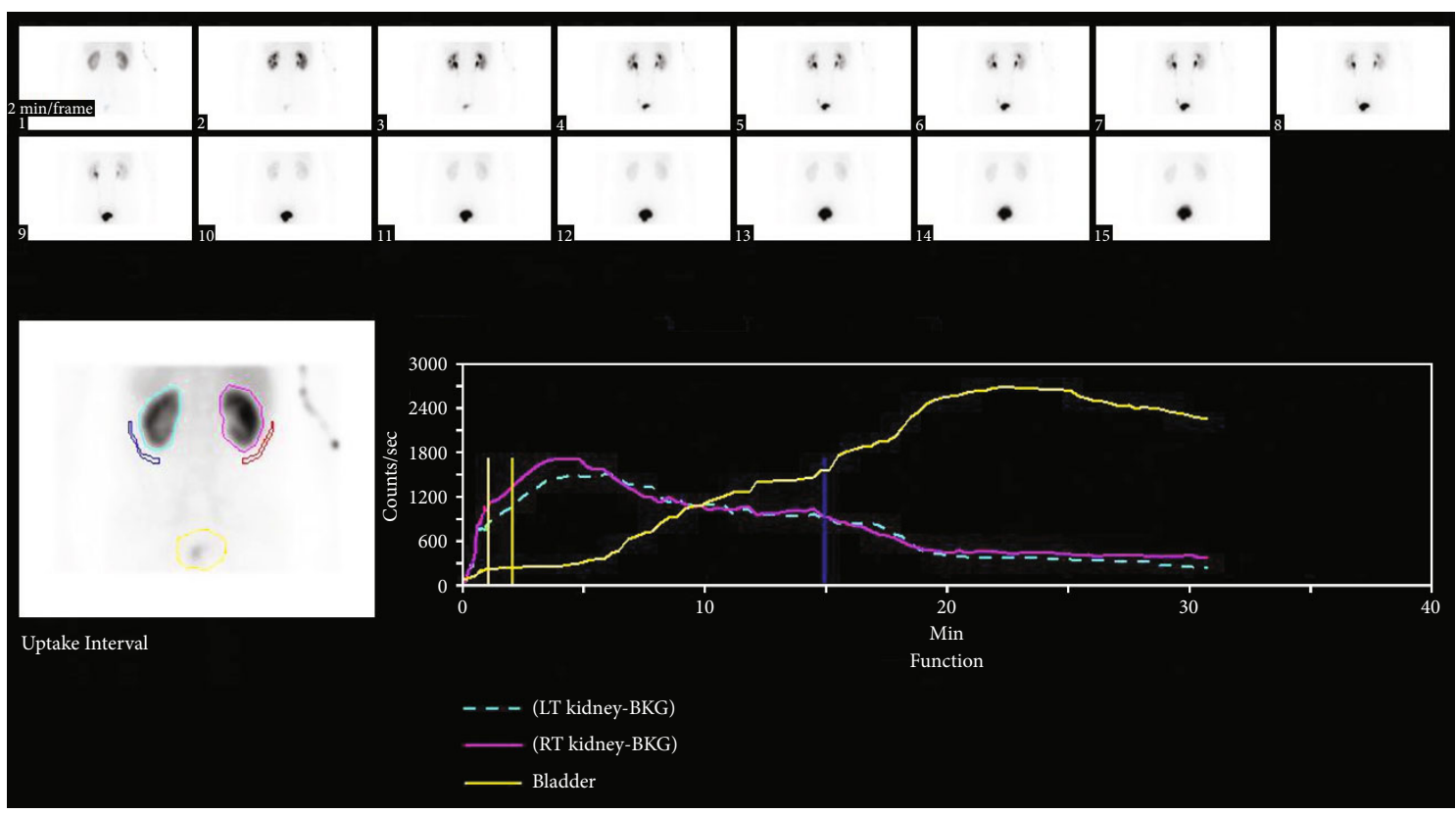

(d)

FIGURE 3: Changes in the $\mathrm{T}_{1 / 2}$ of the diuretic ${ }^{99 \mathrm{~m}}$ Tc-MAG3 scan according to the preoperative $\mathrm{T}_{1 / 2}$ of the diuretic ${ }^{99 \mathrm{~m}} \mathrm{Tc}-\mathrm{MAG} 3$ scan results. (a, b) Preoperative values of the $\mathrm{T}_{1 / 2}$ of the diuretic ${ }^{99 \mathrm{~m}} \mathrm{Tc}-\mathrm{MAG} 3$ scan were 7.0 in the left kidney and 9.3 in the right kidney, which means no delay in the $\mathrm{T}_{1 / 2}$ of the upper urinary tract (a). The values were improved to 4.7 in the left kidney and 5.3 in the right kidney postoperatively (b). (c, d) Preoperative values of the $\mathrm{T}_{1 / 2}$ of the diuretic ${ }^{99 \mathrm{~m}} \mathrm{Tc}-\mathrm{MAG} 3$ scan were 73.3 in the left kidney and 44.0 in the right kidney, which means obvious upper urinary tract obstruction (c). The values were markedly improved to 11.6 in the left kidney and 11.6 in the right kidney postoperatively $(\mathrm{d})$.

IPSS score of 50.17, 82.11, 72.29, and 22.44, respectively, which cautions that the data of this cohort cannot be generalized for all BPH patients. However, the present study showed the importance of bladder function, which was central to the explanation of the probability of CKD progression in BPO patients, and provided a message of the importance of decompression of BPO in the prevention of kidney damage due to the delay in urinary drainage.

\section{Conclusions}

In conclusion, storage-phase bladder dysfunction (low compliance and/or detrusor overactivity) may be a key factor for kidney damage resulting from delayed urinary drainage in patients with BPO. Such a delay could be improved by the decompression of BPO. Based on the results of the present study with supporting literature, the authors suggest that 
TABle 3: Association between postoperative significant proteinuria and other factors in postoperative data.

\begin{tabular}{|c|c|}
\hline Upper tract factor & \\
\hline Potential obstruction $^{1}$ & $0.233, p=0.338$ \\
\hline eGFR & $-0.313, p=0.191$ \\
\hline \multicolumn{2}{|l|}{ Lower tract factor } \\
\hline \multicolumn{2}{|l|}{ Uroflowmetry } \\
\hline Qmax & $-0.093, p=0.706$ \\
\hline Voiding volume & $-0.054, p=0.825$ \\
\hline Postvoid residual volume & $-0.440, p=0.059$ \\
\hline \multicolumn{2}{|l|}{ General factors } \\
\hline Age & $0.118, p=0.630$ \\
\hline Body mass index & $-0.163, p=0.505$ \\
\hline Diabetes mellitus & $0.696, p=0.001^{*}$ \\
\hline Hypertension & $0.420, p=0.073$ \\
\hline
\end{tabular}

the role of $\mathrm{BPH}$ progression in the development of CKD is as follows: " $\mathrm{BPH} \rightarrow \mathrm{BOO} \rightarrow$ storage-phase bladder dysfunction with bladder wall thickness $\rightarrow$ delay in upper urinary tract drainage $\rightarrow$ kidney damage (proteinuria) $\rightarrow$ CKD progression". Therefore, when surgeons encounter patients with severe $\mathrm{BPH}$, they should seriously consider preoperative urodynamic studies to evaluate storage-phase bladder function. Given the cost of diuretic renal scans, surgeons should also perform urinalysis in those patients to detect proteinuria before and after surgery.

\section{Data Availability}

The data used to support the findings of this study are available from the corresponding author upon request.

\section{Ethical Approval}

The institutional review boards of the 5 hospitals approved the observational study design and the access to the patients' medical records (central approval number: VC16OIMI0106). This study was performed in accordance with the Declaration of Helsinki. Written informed consent was obtained from all patients.

\section{Consent}

All patients provided informed consent for all surgical procedures.

\section{Conflicts of Interest}

The authors declare that they have no conflicts of interest.

\section{Acknowledgments}

The authors are particularly grateful to all patients who participated in the whole course of this study. This work was supported by the National Research Foundation of Korea(NRF) grant funded by the Korea government(MSIT) (No. 2019R1C1C1008339).

\section{Supplementary Materials}

. The Exel data used to support the findings of this study have been deposited in the Supplementary file. (Supplementary Materials)

\section{References}

[1] M.-K. Li, L. Garcia, N. Patron et al., "An Asian multinational prospective observational registry of patients with benign prostatic hyperplasia, with a focus on comorbidities, lower urinary tract symptoms and sexual function," BJU International, vol. 101, no. 2, pp. 197-202, 2008.

[2] C. G. Roehrborn, "BPH progression: concept and key learning from MTOPS, ALTESS, COMBAT, and ALF-ONE," BJU International, vol. 101, Supplement 3, pp. 17-21, 2008.

[3] H. Lepor, "Pathophysiology, epidemiology, and natural history of benign prostatic hyperplasia," Reviews in urology, vol. 6, Supplement 9, pp. S3-S10, 2004.

[4] A. D. Rule, M. M. Lieber, and S. J. Jacobsen, "Is benign prostatic hyperplasia a risk factor for chronic renal failure?," The Journal of Urology, vol. 173, no. 3, pp. 691-696, 2005.

[5] A. D. Rule, D. J. Jacobson, R. O. Roberts et al., "The association between benign prostatic hyperplasia and chronic kidney disease in community-dwelling men," Kidney International, vol. 67, no. 6, pp. 2376-2382, 2005.

[6] A. A. Shokeir, A. P. Provoost, and R. J. Nijman, "Recoverability of renal function after relief of chronic partial upper urinary tract obstruction," BJU International, vol. 83, no. 1, pp. 1117, 1999.

[7] A. S. Levey, J. Coresh, T. Greene et al., "Using standardized serum creatinine values in the modification of diet in renal disease study equation for estimating glomerular filtration rate," Annals of Internal Medicine, vol. 145, no. 4, pp. 247-254, 2006.

[8] National Kidney Foundation, "K/DOQI clinical practice guidelines for chronic kidney disease: evaluation, classification, and stratification," American journal of kidney diseases, vol. 39, pp. S1-S266, 2002.

[9] M. Miyake, N. Tanaka, I. Asakawa et al., "Assessment of lower urinary symptom flare with overactive bladder symptom score and International Prostate Symptom Score in patients treated with iodine-125 implant brachytherapy: long-term follow-up experience at a single institute," BMC Urology, vol. 17, no. 1, p. 62, 2017.

[10] P. Abrams, "Bladder outlet obstruction index, bladder contractility index and bladder voiding efficiency: three simple indices to define bladder voiding function," BJU International, vol. 84, no. 1, pp. 14-15, 1999.

[11] J. L. H. R. Bosch, W. C. J. Hop, W. J. Kirkels, and F. H. Schröder, "Natural history of benign prostatic hyperplasia: appropriate case definition and estimation of its prevalence in the community," Urology, vol. 46, no. 3, pp. 34-40, 1995. 
[12] M. H. Blanker, A. M. Bohnen, F. P. Groeneveld, R. M. Bernsen, A. Prins, and J. L. H. R. Bosch, "Normal voiding patterns and determinants of increased diurnal and nocturnal voiding frequency in elderly men," The Journal of Urology, vol. 164, no. 4, pp. 1201-1205, 2000.

[13] D. S. Lee, S. W. Kim, and D. W. Sohn, "Association Between Nocturnal Frequency and Erectile Function in Eugonadal Men With Benign Prostatic Obstruction: A Cross Sectional Study," The World Journal of Men's Health, vol. 38, 2020.

[14] W. Y. Lin, S. P. Changlai, and C. H. Kao, "Normal ranges of renal physiological parameters for technetium-99m mercaptoacetyltriglycine and the influence of age and sex using a camera-based method," Urologia Internationalis, vol. 60, no. 1, pp. 11-16, 1998.

[15] K. Matsuoka, S. Iida, K. Tomiyasu, A. Shimada, S. Suekane, and S. Noda, "Holmium laser resection of the prostate," Journal of Endourology, vol. 12, no. 3, pp. 279-282, 1998.

[16] R. Prakash, A. Puri, R. Anand, A. K. Jain, B. Lal, and V. Garg, "Predictors of upper tract damage in pediatric neurogenic bladder," Journal of Pediatric Urology, vol. 13, no. 5, pp. 503.e1-503.e7, 2017.

[17] F. Presicce, C. De Nunzio, M. Gacci, E. Finazzi Agrò, and A. Tubaro, "Non-invasive ultrasound measurements in male patients with LUTS and benign prostatic obstruction: implication for diagnosis and treatment," minerva urol nefrol journal, vol. 69, no. 3, pp. 220-233, 2017.

[18] C. De Nunzio, F. Presicce, R. Lombardo, S. Carter, C. Vicentini, and A. Tubaro, "Detrusor overactivity increases bladder wall thickness in male patients: a urodynamic multicenter cohort study," Neurourology and Urodynamics, vol. 36, no. 6, pp. 1616-1621, 2017.

[19] A.-F. Ahmed and M. Bedewi, "Can bladder and prostate sonomorphology be used for detecting bladder outlet obstruction in patients with symptomatic benign prostatic hyperplasia?," Urology, vol. 98, pp. 126-131, 2016.

[20] R. G. Gerridzen, A. M. Thijssen, and E. Dehoux, "Risk factors for upper tract deterioration in chronic spinal cord injury patients," The Journal of Urology, vol. 147, no. 2, pp. 416418, 1992.

[21] D.-H. Zermann, U. Löffler, O. Reichelt, H. Wunderlich, S. Wilhelm, and J. Schubert, "Bladder dysfunction and end stage renal disease," International Urology and Nephrology, vol. 35, no. 1, pp. 93-97, 2003.

[22] J. Abarbanel and E.-L. Marcus, "Impaired detrusor contractility in community-dwelling elderly presenting with lower urinary tract symptoms," Urology, vol. 69, no. 3, pp. 436-440, 2007.

[23] A. Gammie, M. Kaper, C. Dorrepaal, T. Kos, and P. Abrams, "Signs and symptoms of detrusor underactivity: an analysis of clinical presentation and urodynamic tests from a large group of patients undergoing pressure flow studies," European Urology, vol. 69, no. 2, pp. 361-369, 2016.

[24] S. Juma, "Urinary retention in women," Current Opinion in Urology, vol. 24, no. 4, pp. 375-379, 2014.

[25] J. Stoffel, D. Lightner, A. Peterson, J. Sandhu, A. Suskind, and J. Wei, "Non-Neurogenic Chronic Urinary Retention: Consensus Definition, Management Strategies, and Future Opportunities," Dec 2019 Available from URL https://www.auanet .org/guidelines/chronic-urinary-retention.

[26] C. L. A. Negro and G. H. Muir, "Chronic urinary retention in men: how we define it, and how does it affect treatment out- come," BJU International, vol. 110, no. 11, pp. 1590-1594, 2012.

[27] S. K. Hong, S. T. Lee, S. J. Jeong et al., "Chronic kidney disease among men with lower urinary tract symptoms due to benign prostatic hyperplasia," BJU International, vol. 105, no. 10, pp. 1424-1428, 2010.

[28] D. S. Lee, H. W. Kim, and S.-J. Lee, “Transurethral prostate surgery as a preventive method against progression of chronic kidney disease in patients with urodynamically proven bladder outlet obstruction," World Journal of Urology, 2019.

[29] K. M. Andrassy, "Comments on 'KDIGO 2012 clinical practice guideline for the evaluation and management of chronic kidney disease'," Kidney International, vol. 84, no. 3, pp. 622623, 2013.

[30] A. S. Levey, L. A. Stevens, and J. Coresh, "Conceptual model of CKD: applications and implications," American Journal of Kidney Diseases, vol. 53, no. 3, pp. S4-S16, 2009.

[31] H. Nomura, N. Seki, A. Yamaguchi, and S. Naito, "Comparison of photoselective vaporization and standard transurethral resection of the prostate on urodynamics in patients with benign prostatic hyperplasia," International Journal of Urology, vol. 16, no. 8, pp. 657-662, 2009.

[32] M. Tkocz and A. Prajsner, "Comparison of long-term results of transurethral incision of the prostate with transurethral resection of the prostate, in patients with benign prostatic hypertrophy," Neurourology and Urodynamics, vol. 21, no. 2, pp. 112-116, 2002.

[33] E. Stoner, "Three-year safety and efficacy data on the use of finasteride in the treatment of benign prostatic hyperplasia," Urology, vol. 43, no. 3, pp. 284-294, 1994.

[34] C. G. Roehrborn, P. Siami, J. Barkin et al., "The influence of baseline parameters on changes in international prostate symptom score with dutasteride, tamsulosin, and combination therapy among men with symptomatic benign prostatic hyperplasia and an enlarged prostate: 2 -year data from the CombAT study," European Urology, vol. 55, no. 2, pp. 461471, 2009. 